\title{
Erratum: Gravitational-Wave Signature of a First-Order Quantum Chromodynamics Phase Transition in Core-Collapse Supernovae [Phys. Rev. Lett. 125, 051102 (2020)]
}

Shuai Zha $\odot$, Evan P. O’Connor, Ming-chung Chu, Lap-Ming Lin, and Sean M. Couch

Q (Received 14 October 2021; published 15 November 2021)

DOI: 10.1103/PhysRevLett.127.219901

In Fig. 4 and the right panel of Fig. S3 of this Letter, we were plotting $h_{\text {char }} / \sqrt{f}$ with a source distance of 1 kpc. Below, we provide the corrected Fig. 4 that plots $h_{\text {char }}$ with a source distance of $10 \mathrm{kpc}$. The affected Fig. S3 in the Supplemental Material has been replaced with a corrected one. They have no conflict with the text in this Letter and do not affect our conclusions.

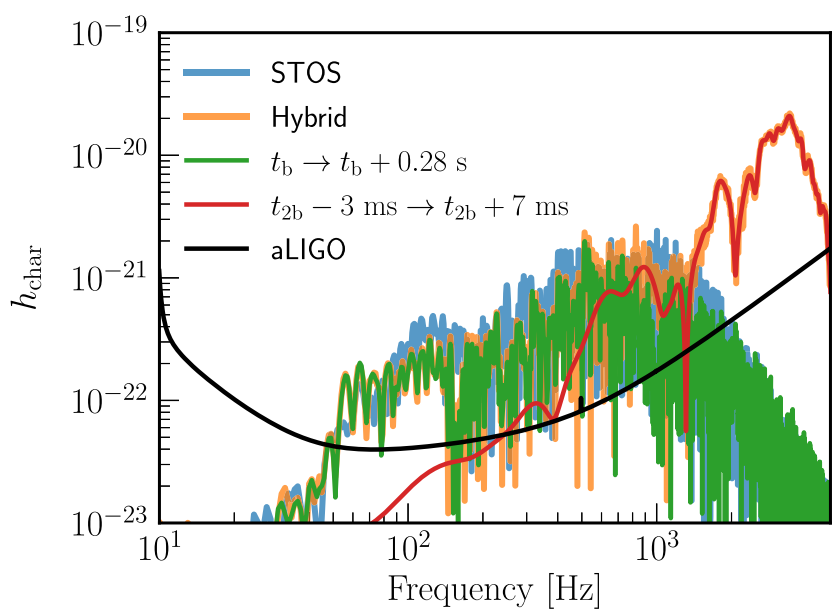

FIG. 4. Dimension-less characteristic strain spectra $\left[h_{\text {char }}(f)\right]$ of the GW signals from $t_{b}$ to $t_{b}+400$ ms with the STOS (blue) and hybrid (orange) EOSs. Also shown are the $h_{\text {char }}(f)$ of the GW signal for the hybrid EOS in the time interval of from $t_{b}$ to $t_{b}+280$ ms (green) and between $t_{2 b}-3 \mathrm{~ms}$ and $t_{2 b}+7 \mathrm{~ms}$ (red). The black line is the sensitivity spectrum of advanced LIGO [38].

[38] L. Barsotti, P. Fritschel, M. Evans, and S. Gras (LIGO Collaboration), Technical Report LIGO-T1800044-v5, 2018, https://dcc .ligo.org/LIGO-T1800044/public.

Published by the American Physical Society under the terms of the Creative Commons Attribution 4.0 International license. Further distribution of this work must maintain attribution to the author(s) and the published articles title, journal citation, and DOI. 